\title{
Lentiviral-mediated Shh reverses the adverse effects of high glucose on osteoblast function and promotes bone formation via Sonic hedgehog signaling
}

\author{
ZHU-LING JIANG ${ }^{1}$, HAN JIN ${ }^{2}$, ZHONG-SHUANG LIU ${ }^{2}$, MING-YUE LIU ${ }^{3}$, XIAO-FANG CAO ${ }^{3}$,

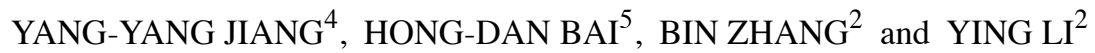

${ }^{1}$ Department of Implantology, ${ }^{2}$ Institute of Hard Tissue Development and Regeneration, ${ }^{3}$ Department of Dentistry,

The Second Affiliated Hospital, Harbin Medical University; ${ }^{4}$ Department of Dentistry, The Affiliated Hospital, Harbin Institute of Technology, Harbin, Heilongjiang 150001; ${ }^{5}$ Feiyang Dental Clinic, Heihe, Heilongjiang 164300, P.R. China

Received November 5, 2018; Accepted July 11, 2019

DOI: $10.3892 / \mathrm{mmr} .2019 .10540$

\begin{abstract}
Patients with diabetes tend to have an increased incidence of osteoporosis, which may be associated with hyperglycemia; however, the pathogenic mechanisms governing this interaction remain unknown. The present study sought to investigate whether elevated extracellular glucose levels of bone mesenchymal stem cells (BMSCs) could influence osteoblastic differentiation and whether the intracellular Sonic hedgehog (Shh) pathway could adjust the effects. Furthermore, to verify the results in vivo, a rat tooth extraction model was constructed. BMSCs were incubated in eight types of culture medium, including low glucose (LG), LG + lentivirus (Lenti), LG + Lenti-small interfering RNA (Lenti-siRNA), LG + Lenti-Shh, high glucose (HG), HG + Lenti, HG + Lenti-siRNA and HG + Lenti-Shh. The lentiviral transfection efficiency was observed using a fluorescence microscope; protein and mRNA expression was detected by western blotting and reverse transcription-quantitative polymerase chain reaction (RT-qPCR). The matrix mineralization and alkaline phosphatase (ALP) activity of BMSCs were examined by Alizarin red staining and ALP activity assays, respectively. The expression of osteogenesis-related genes in BMSCs were quantified by RT-qPCR. The alveolar ridge reduction was measured and histological sections were used to evaluate new bone formation in the tooth socket. With high concentrations of glucose, Shh expression, matrix mineralization nodules formation, ALP activity and the levels of bone
\end{abstract}

Correspondence to: Dr Bin Zhang or Dr Ying Li, Institute of Hard Tissue Development and Regeneration, The Second Affiliated Hospital, Harbin Medical University, 246 Xuefu Road, Harbin, Heilongjiang 150001, P.R. China

E-mail: zhangbin@hrbmu.edu.cn

E-mail: bonnieli1122@gmail.com

Key words: bone marrow stromal cells, sonic hedgehog, diabetes, osteogenic differentiation, osteogenesis, lentiviral vector morphogenic protein 4 (BMP4), bone sialoprotein (BSP) and osteopontin (OPN) expression were greatly reduced compared with LG and corresponding control groups. Whereas activated Shh signaling via Lenti-Shh could increase the number of matrix mineralization nodules, ALP activity, and the expression levels of BMP4, BSP and OPN in BMSCs. Additionally, in vivo assays demonstrated that Lenti-Shh induced additional bone formation. Collectively, the results of the present study indicated that HG inhibited the Shh pathway in osteoblasts and resulted in patterning defects during osteoblastic differentiation and bone formation, while the activation of Shh signaling could suppress these deleterious effects.

\section{Introduction}

Diabetes mellitus (DM) is a metabolic disease that is characterized by hyperglycemia (1). DM has multiple possible long-term complications, including vascular lesions, neuropathy, retinopathy, nephropathy and bone osteoporosis. DM was determined to affects the skeletal system (2). Females with DM have a three-fold higher risk of fracture when compared with non-diabetic females (3). In addition, fracture patients with diabetes require longer hospitalization times than those without diabetes (4); however, the underlying mechanisms associated with fracture risk in diabetes are yet to be completely elucidated. Evidence has also demonstrated that DM accelerates alveolar bone resorption (5). In our previous study, rats subjected to experimental DM exhibited more serious alveolar bone destruction when compared with the normal group (6). This also suggested that type 2 DM (T2DM) elevated the levels of tumor necrosis factor- $\alpha$, interleukin- $1 \beta$ and lipopolysaccharide in the gingival crevicular fluid of rats (6). The enhanced levels of inflammatory mediators contribute to an increase in osteoblast apoptosis, decreasing their abundance (7). Decreased activity of osteoblasts under diabetic conditions has been discussed in numerous studies in both humans and animal models $(8,9)$; however, despite numerous studies that have addressed this problem, the precise molecular mechanism underling the effects of glucose in altering bone formation and osteoblastic differentiation is yet to be elucidated. 
Lentiviral vector technology has now become an effective tool for therapeutic gene delivery (10-12). Effectively, lentiviral vectors can infect dividing and non-dividing cells then integrate exogenous genes or exogenous short hairpin RNAs into a host chromosome, which culminates in the stable and long-term expression of the target genes (13).

Hedgehog (Hh) signals serve an important role in evolution and numerous biological events (14). The Hh gene family comprises at least three members, including Sonic hedgehog (Shh), Desert hedgehog and Indian hedgehog. Shh is involved in the formation of organs and tissues; Shh proteins are involved in the same signaling pathway. The activated Shh ligand binds to the transmembrane protein receptor protein patched homolog $1(\mathrm{PTCH})$, which results in the release of the downstream protein smoothened (SMO). SMO subsequently transduces signaling downstream which in turn activates the transcription factors glioma-associated homologs. Phosphorylated Gli enters the nucleus and activates target genes (14). Without the Shh ligand, PTCH binds to SMO and suppresses the activation of SMO, as well as the associated genes (15). The Shh pathway is involved in multiple developmental processes by regulating cell differentiation (16). A previous study reported that Shh induces osteoblastic differentiation and bone formation as evidenced by an in vitro culture system and in vivo transplantation experiments (17). Additionally, it was demonstrated that by adding Shh to bone mesenchymal stem cells (BMSCs), the differentiation of uncommitted BMSCs to the osteoblast lineage was promoted (18). A previous study revealed that the altered expression of Shh and bone morphogenic protein 4 (BMP4), and their downstream genes are associated with variations of the telencephalon in the embryos of diabetic mice (19). In addition, the study of Dunaeva et al (20) demonstrated that diabetes is associated with a functional inhibition of the Shh signaling pathway.

These studies indicated that the Shh signaling pathway was associated with osteoblastic differentiation and DM, while DM could be associated with the skeletal system and osteoblastic activity. We hypothesized that high glucose (HG) levels altered the Shh signaling pathway in osteoblasts and affected osteoblast functions. Therefore, in the present study, the Shh pathway was induced and suppressed vial entiviral vector-mediated Shh/small interfering (si)RNA gene transfer in vitro; we also investigated whether in an in vivo animal model, whether alterations in Shh expression may alter the effects of HG on osteoblastic differentiation of BMSCs and new bone formation.

\section{Materials and methods}

Statement of ethics. All animal experiments were conducted in strict accordance with the principles of medical ethics and were approved by Animal Care and Use Committee of The Second Affiliated Hospital of Harbin Medical University (Harbin, China). All necessary permits were obtained for the described field studies.

Isolation and culture of BMSCs. BMSCs were isolated from 3 -week-old male Sprague-Dawley rats $(\mathrm{n}=30$, average weight of 80-100 g), which were purchased from the Department of the Animal Experiment Center of The Second Affiliated Hospital of Harbin Medical University. Their housing conditions were as follows: $18-26^{\circ} \mathrm{C}$, humidity $40-70 \%$, fresh air, $12 \mathrm{~h} \mathrm{light/dark} \mathrm{cycle,} \mathrm{and} \mathrm{free} \mathrm{access} \mathrm{to} \mathrm{food} \mathrm{and} \mathrm{water.} \mathrm{Briefly,}$ rats were sacrificed, soaked in $75 \%$ ethanol for $10 \mathrm{~min}$, and the femurs and tibias were isolated from the soft tissues. The proximal and distal ends of the femur and tibia were removed. The exposed bone marrow cavity was flushed with $30 \mathrm{ml}$ of Dulbecco's modified Eagle's medium (DMEM; Hyclone; GE Healthcare Life Sciences) containing 10\% fetal bovine serum (FBS; Hyclone; GE Healthcare Life Sciences), $100 \mathrm{U} / \mathrm{ml}$ penicillin and $100 \mu \mathrm{g} / \mathrm{ml}$ streptomycin (Sigma-Aldrich, Merck $\mathrm{KGaA}$ ). The cell suspension was obtained by repeated rinsing through a 5-gauge needle, and was placed in a centrifuge tube. The supernatant was discarded after centrifugation at $1,000 \mathrm{x} \mathrm{g}$ for $10 \mathrm{~min}$ at room temperature. Cells were then seeded at a density of $2 \times 10^{6}$ cells $/ \mathrm{ml}$ in a $25 \mathrm{~cm}^{2}$ plastic culture flask in the low glucose (LG)-DMEM containing 10\% FBS, $100 \mathrm{U} / \mathrm{ml}$ penicillin and $100 \mu \mathrm{g} / \mathrm{ml}$ streptomycin, and incubated in $5 \% \mathrm{CO}_{2}$ at $37^{\circ} \mathrm{C}$. Following a 72 -h incubation, the unattached cells were removed by replacing the medium and the remaining cells were considered to be BMSCs. When the cells reached $90 \%$ confluence, they were trypsinized by $0.25 \%$ trypsin and $0.02 \%$ ethylenediamine tetraacetic acid (Gibco; Thermo Fisher Scientific, Inc.) for $2 \mathrm{~min}$, and then passaged at a density of $10^{2}$ per $\mathrm{cm}^{2}$. The first-passage BMSCs were cultured in $10 \% \mathrm{FBS}, 100 \mathrm{U} / \mathrm{ml}$ penicillin and $100 \mu \mathrm{g} / \mathrm{ml}$ streptomycin with DMEM containing different concentrations of glucose, $5 \% \mathrm{CO}_{2}$ at $37^{\circ} \mathrm{C}$ (LG-DMEM, $1,000 \mathrm{mg} / 1$ glucose; HG-DMEM, 4,500 mg/l glucose). The third passage generation cells were used for further studies.

Transduction with lentiviral vectors. Lentivirus gene transfer vectors fused with a green fluorescent protein (GFP) sequence were constructed by Shanghai GenePharma Co.,Ltd.Lentiviral vectors can infect BMSCs then integrate RNAs into a host chromosome. The targeting sequences for Rattus norvegicus Shh (NM-017221) were 5'-GGTGCCAAGAAGGTCTTC TAC-3', and the titer of Lenti-Shh (activate Shh signaling), Lenti-siRNA (inhibit Shh signaling) and Lenti (negative control) were all $1 \times 10^{8} \mathrm{UT} / \mathrm{ml}$. The third passage of BMSCs was plated in 24 -well plates at a density of $0.5 \times 10^{5}$ cells/well in $0.5 \mathrm{ml}$ medium per well prior to transduction. Following a $24-\mathrm{h}$ incubation the cells had grown to $30-50 \%$ confluence; $10 \% \mathrm{FBS}, 100 \mathrm{U} / \mathrm{ml}$ penicillin and $100 \mu \mathrm{g} / \mathrm{ml}$ streptomycin with the differing DMEM were removed and transductions were performed at a multiplicity of infection (MOI) of 10 according to a preliminary experiment. BMSCs were incubated in 10\% FBS-DMEM under eight different conditions: LG + Lenti-Shh, LG + Lenti-siRNA, LG, LG + Lenti, $\mathrm{HG}+$ Lenti-Shh, HG + Lenti-siRNA, HG and HG + Lenti. The volume of fresh medium was $0.5 \mathrm{ml}$ per well. On the third day, the culture medium was replaced with 10\% FBS LG-DMEM or $10 \%$ FBS HG-DMEM (1 ml/well), respectively. Every other day medium was replenished, and it was suitable to proceed experiments until day 6 . The transduction efficiency and the morphology of the BMSCs was evaluated by two researchers independently via fluorescence and phase contrast microscopes (Olympus Corporation) in 5-7 fields per view (magnification, x100). 
Western blot analysis. Following transduction, cells were cultured in the eight different types of media for 6 days, and were then lysed in ice-cold radioimmunoprecipitation assay (Santa Cruz Biotechnology, Inc.) and phenylmethyl sulfonyl fluoride lysis buffer (Santa Cruz Biotechnology, Inc.) for 2 sec. The cells were subsequently scraped from the plate with a cell scraper, and transferred to a $1.5 \mathrm{ml}$ microcentrifuge tube. The lysates were separated by centrifugation at $12,000 \mathrm{xg}$ for $5 \mathrm{~min}$ at $4^{\circ} \mathrm{C}$. The supernatants were collected and stored at $-80^{\circ} \mathrm{C}$ until western blotting was performed. The protein concentration was quantified with a bicinchoninic acid (BCA) protein assay kit (Beyotime Institute of Biotechnology). The samples were separated by $12 \%$ SDS-PAGE based on the molecular weight of the target proteins (Shh, 19.45), and were then transferred to a polyvinylidene difluoride membrane (EMD Millipore). After blocking with 5\% non-fat milk in TBST and $1 \mathrm{X}$ Tris-buffered saline with $0.1 \%$ Tween-20, the membranes were incubated with the following antibodies: Shh (C9C5) rabbit monoclonal antibody (dilution 1:1,000; cat. no. 2207, Cell Signaling Technology, Inc.) and anti- $\beta$-actin (dilution 1:1,000; cat. no. 03-102, Sigma-Aldrich; Merck KGaA) at $4^{\circ} \mathrm{C}$ overnight. The membranes were then incubated with horseradish peroxidase-conjugated AffiniPure Goat Anti-Mouse IgG $(\mathrm{H}+\mathrm{L})$, according to the origin of the primary antibodies, (dilution 1:5,000; cat. no. ZB-2305, OriGene Technologies, Inc.) for $1 \mathrm{~h}$ at room temperature and were detected by using enhanced chemiluminescence plus reagents (GE Healthcare Life Sciences) according to the manufacturer's protocols; the emitted light was captured on an X-ray film.

Alizarin red staining. The mineralization of the third passage BMSCs was determined using Alizarin red staining (Sigma-Aldrich, Merck KGaA). BMSCs were seeded in 24-well plates at a density of $5.0 \times 10^{4}$ cells/well. Following transduction, the eight different media types were removed and replaced with osteogenic medium containing $50 \mu \mathrm{g} / \mathrm{ml}$ ascorbic acid (Sigma-Aldrich; Merck KGaA), 10 mmol/1 $\beta$-glycerophosphate sodium (Sigma-Aldrich; Merck KGaA), and 10\% FBS LG-DMEM or $10 \%$ FBS HG-DMEM. The culture medium was replaced every other day and staining was performed after three weeks (21 days later, osteogenic-inducing medium can promote the formation of mineralized nodules). Cells were fixed with $70 \%$ ethanol for $1 \mathrm{~h}$ at room temperature, washed with PBS twice per well and stained with $40 \mathrm{mM}$ Alizarin red ( $\mathrm{pH} 4.2$, Sigma-Aldrich; Merck KGaA) for $15 \mathrm{~min}$ at room temperature. The stained cells were rinsed with $\mathrm{dH}_{2} \mathrm{O}$ four times to eliminate nonspecific staining, and then observed using a light microscope (Nikon Corporation). We randomly selected 5 fields of vision per sample for analysis (magnification, x100).

Alkaline phosphatase (ALP) activity assay. Following transduction, the third passage cells in 24-well plates were treated with the osteogenic medium as described above. The culture medium was changed every 2 days. Following 2 weeks of incubation in $5 \% \mathrm{CO}_{2}$ at $37^{\circ} \mathrm{C}$, ALP activity of the BMSCs was detected using an ALP assay kit (Nanjing KeyGen Biotech Co., Ltd.) according to the manufacturer's protocol. The absorbance was recorded using a microplate absorbance reader (Bio-Rad Laboratories, Inc.; Model-680) at $405 \mathrm{~nm}$. The protein concentration was measured with a BCA protein assay kit (Beyotime Institute of Biotechnology). The ALP activity was normalized to the total protein content.

Reverse transcription-quantitative polymerase chain reaction $(R T-q P C R)$. RNA samples for eight groups were analyzed to measure the effects of high glucose on the expression of osteogenesis-related genes in BMSCs. RT-qPCR was also used to quantify the expression levels of the seven-transmembrane protein SMO. On day 6 following transduction, total RNA was extracted using TRIzol ${ }^{\circledR}$ reagent (Invitrogen; Thermo Fisher Scientific, Inc.), and the concentration of total RNA was measured with an ultraviolet spectrophotometer (Thermo Fisher Scientific, Inc.). Total RNA (300 ng) was reverse transcribed into cDNA using a Prime Script $^{\mathrm{TM}}$ RT Reagent kit (Takara Bio, Inc.) and RT was performed at $44^{\circ} \mathrm{C}$ for $60 \mathrm{~min}$. Then, the mixture was incubated at $95^{\circ} \mathrm{C}$ for $5 \mathrm{~min}$. All of the primers for PCR are listed in Table I. qPCR was performed on a CFX-96 machine (Bio-Rad Laboratories, Inc.) using a SYBR PrimeScript EX Taq PCR (Takara Bio, Inc.) according to the manufacturer's protocols. All reactions were conducted in a $20 \mu 1$ mixture containing cDNA preparation (1 $\mu 1$ ), $10 \mu \mathrm{S} \mathrm{SYBR}^{\circledR}$ Premix Ex Taq ${ }^{\mathrm{TM}}$ (Tli RNaseH Plus), $1 \mu \mathrm{l}$ of each primer and $7 \mu \mathrm{l}$ diethylpyrocarbonate water. The thermocycling conditions were $95^{\circ} \mathrm{C}$ for $10 \mathrm{~min}$, and then 40 cycles of $95^{\circ} \mathrm{C}$ for $20 \mathrm{sec}$ and $60^{\circ} \mathrm{C}$ for $30 \mathrm{sec}$. A melting curve was acquired using $95^{\circ} \mathrm{C}$ for $15 \mathrm{sec}, 60^{\circ} \mathrm{C}$ for $30 \mathrm{sec}$, and $95^{\circ} \mathrm{C}$ for $15 \mathrm{sec}$. The expression levels were normalized to the expression of the housekeeping gene $\beta$-actin. Data were analyzed using the $2^{-\Delta \Delta \mathrm{Cq}}$ method (21).

Animal experiments. A total of 60, 3-month-old male Sprague-Dawley rats with average weight of 200-220 g, were obtained from the Department of the Animal Experiment Center of The Second Affiliated Hospital of Harbin Medical University. Their housing conditions were as follows: $18-26^{\circ} \mathrm{C}$, humidity $40-70 \%$, fresh air, $12 \mathrm{~h}$ light/dark cycle with free access to food and water. All animals were acclimatized for at least 1 week. A total of 30 rats were fed an adequate high fat (HF) diet (22) (34.5\% fat, $17.5 \%$ protein, $48 \%$ carbohydrate; Beijing HFK Bio-Technology), while the remaining animals $(n=30)$ received normal chow for 4 weeks. Then, the 30 rats on the HF diet were intraperitoneally injected with streptozotocin (STZ; $30 \mathrm{mg} / \mathrm{kg}$, Sigma-Aldrich; Merck KGaA) dissolved in $0.1 \mathrm{~mol} / 1$ citrate buffer, $\mathrm{pH} 4.5$, to induce models of diabetes. A diagnosis of diabetes was confirmed by measurement of blood glucose randomly on the third and seventh days following STZ administration. Rats were considered diabetic when the random blood glucose levels were $>11.1 \mathrm{mmol} / 1$ (23).

Rat tooth extraction model. The left incisors of the mandibular of 60 rats were cut at the gingival level with a small diamond bur under anesthesia (10\% chloral hydrate, $300 \mathrm{mg} / \mathrm{kg}$ animal bodyweight, intraperitoneal injection) at days $10,8,6,4$ or 2 following the induction of diabetes; before this, mandibular incisor was fully extracted. On day 0 , the left incisors of the 60 animals were carefully extracted $(24,25)$. The animals were divided into four groups: A normal group as a control 
Table I. Primer sequences employed reverse transcription-quantitative polymerase chain reaction.

\begin{tabular}{llcc}
\hline Genes & \multicolumn{1}{c}{ Sequences (5'-3') } & Size (bp) & Accession no. \\
\hline$\beta$-actin & F: CCCATCTATGAGGGTTACGC & 207 & NM_031144 \\
BSP & $\begin{array}{l}\text { R: TTTAATGTCACGCACGATTTC } \\
\text { F: GATGAAAATGAGCAGGTCGTC } \\
\text { R: GCTTCTTCTCCGTTGTCTCCT }\end{array}$ & 95 & NM_012587 \\
OPN & F: AGGACAGCAACGGGAAGAC & 241 & M99252 \\
BMP4 & R: TGAAACTCGTGGCTCTGATGT & & \\
FMO $:$ GCTCTGCTTTTCGTTTCTTCTT & R: TCCAGTAGTCGTGTGATGAGGT & 199 & NM_012827 \\
& F: TGATGGCTGGAGTAGTGTGGT & & \\
& R: TGAGCAGGTGGAAATAGGATG & 117 & NM_012807
\end{tabular}

F, forward; R, reverse; BMP4, bone morphogenic protein 4; BSP, bone sialoprotein; OPN, osteopontin; SMO, smoothened.
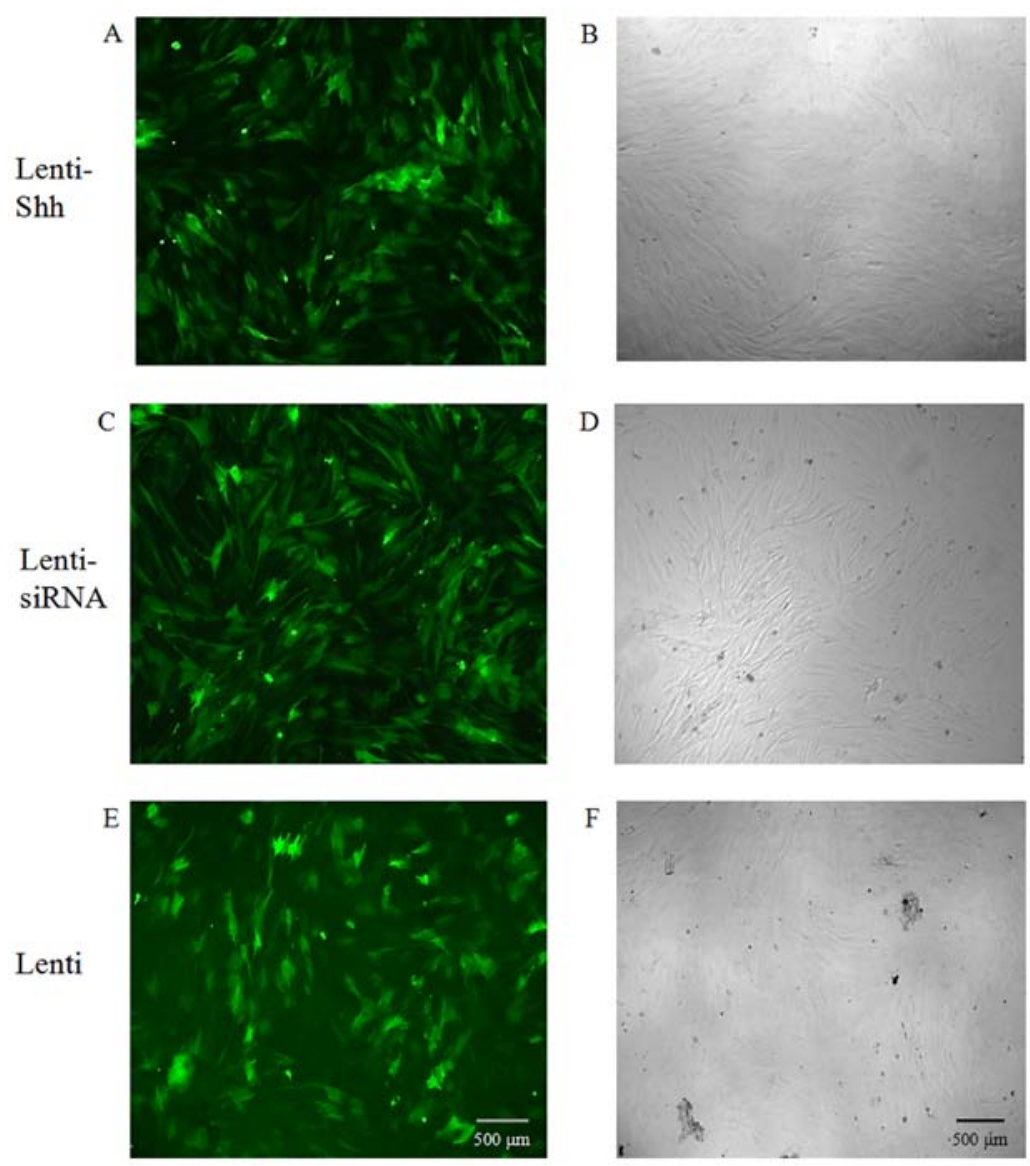

Figure 1. Microscopy analysis of transduced cells. Rat BMSCs were transduced with (A and B) Lenti-Shh, (C and D) Lenti-small interfering RNA and (E and F) Lenti. Fluorescence and phase contrast microscopy analyses to investigate transduction and the morphology of the BMSCs. Scale bar, $500 \mu \mathrm{m}$. BMSCs, bone marrow stromal stem cells; Lenti, lentivirus; Shh, Sonic hedgehog.

(untreated post incisor extraction, $\mathrm{n}=15$ ), a normal group with MOI=10, $50 \mu$ l Lenti-Shh into the tooth socket $(\mathrm{n}=15)$, a DM group with $\mathrm{MOI}=10,50 \mu 1$ Lenti-Shh into the tooth socket $(n=15)$, and a DM group (untreated post incisor extraction, $\mathrm{n}=15$ ). Microliter syringes (Shanghai GAOGE Industrial and Trading Co., Ltd.) were used to inject Lenti-Shh into the tooth socket. The extraction sockets of the teeth were closed with periodontal dressing paste.
The rats were sacrificed at 8 weeks following tooth extraction; the mandible was dissected and the samples were fixed in $10 \%$ neutral buffered formalin at room temperature for 2 days. Soft X-ray images of the mandibles were obtained via cabinet X-ray systems (Faxitron, MC-20). To determine the alveolar ridge reduction after tooth extraction, the distances between the highest point of the mesial at the first molar to the lingual alveolar ridge margin of the incisor were measured 

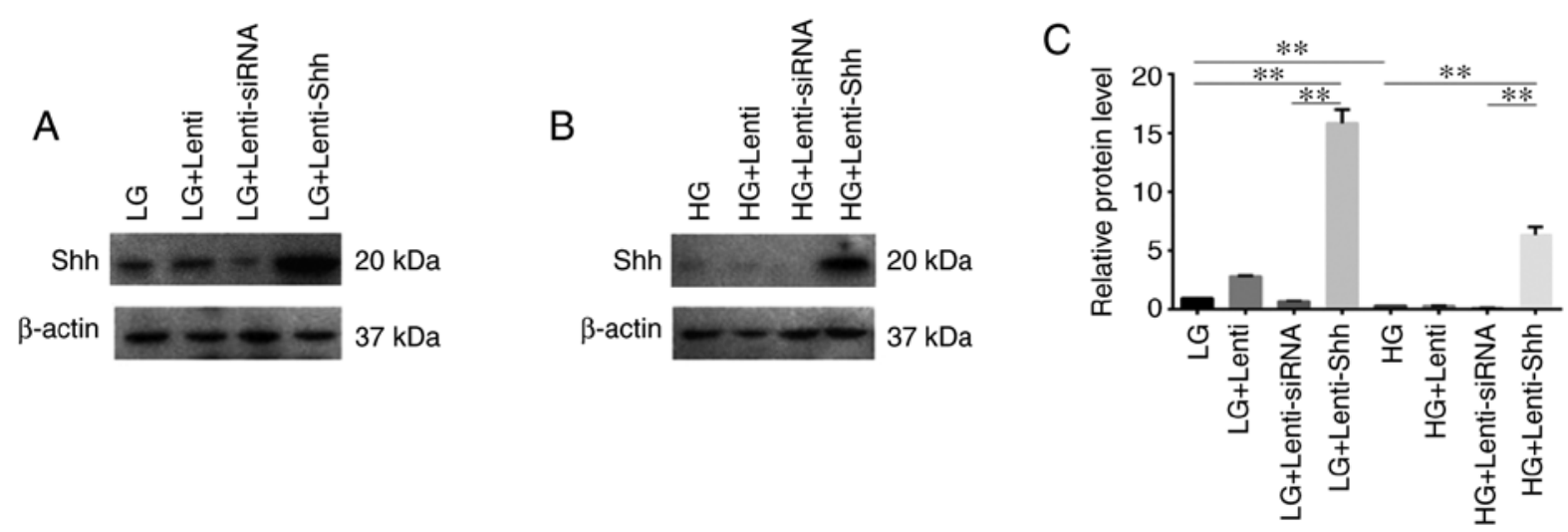

Figure 2. Efficiency of lentiviral-mediated suppression or overexpression of Shh in BMSCs with LG-DMEM or HG-DMEM. (A and B) Expression of Shh protein and (C) relative protein level of Shh in bone marrow stromal stem cells cultured in eight different media conditions: LG, LG + Lenti, LG + Lenti-siRNA, LG + Lenti-Shh, HG, HG + Lenti, HG + Lenti-siRNA and HG + Lenti-Shh. ${ }^{* *} \mathrm{P}<0.01$. HG, high glucose; LG, low glucose; Lenti, lentivirus; Shh, Sonic hedgehog; siRNA, small interfering RNA.

by an image analyzing software (ImageJ; v2.1.4.7, National Institutes of Health) as described previously (26). The reduction rate was calculated according to the following formula: $(1-\mathrm{a} / \mathrm{b}) \times 100 \%$ (a, the length after incisor extraction; $\mathrm{b}$, the length before incisor extraction). Subsequently, the left half of the mandible was decalcified, specimens were decalcified in $10 \%$ ethylene diamine tetraacetic acid for 4 weeks, dehydrated and then embedded in paraffin. The paraffin serial sections in $6 \mu \mathrm{m}$ were obtained in the buccal and lingual direction and stained with hematoxylin and eosin (HE) staining at room temperature for $1 \mathrm{~h}$. The histological sections were used to evaluate new bone formation of the tooth socket in five randomly selected fields per view. Histologic evaluation was performed at 100 magnification using a light microscope (BX50, Olympus Corporation).

Statistical analysis. Statistical analyses were performed using Microsoft Excel and SPSS 23.0 software (IBM Corp.). All of the data are presented as the mean \pm standard deviation. Statistical analysis for differences between multiple groups was conducted using one-way analysis of variance followed by a Tukey's post hoc test for multiple comparisons. The experiments were conducted in triplicate. $\mathrm{P}<0.05$ was considered to indicate a statistically significant difference.

\section{Results}

Transfection efficiency. As the lentiviral constructs were tagged with the GFP reporter, the infection efficiency was examined using fluorescence microscopy and phase contrast microscopy. At $72 \mathrm{~h}$ following transduction, the BMSCs expressed GFP, suggesting that the lentivirus had integrated into the genome of the BMSCs and the infection efficiency was $\sim 80 \%$ when the MOI was 10 (Fig. 1).

Efficiency of lentiviral-mediated suppression or overexpression of Shh in BMSCs with LG-DMEM or HG-DMEM. The present study performed western blotting in order to determine the expression of Shh under LG-DMEM and HG-DMEM treatment following transduction with Lenti-Shh (activate Shh signaling), Lenti-siRNA (inhibit Shh signaling) and Lenti

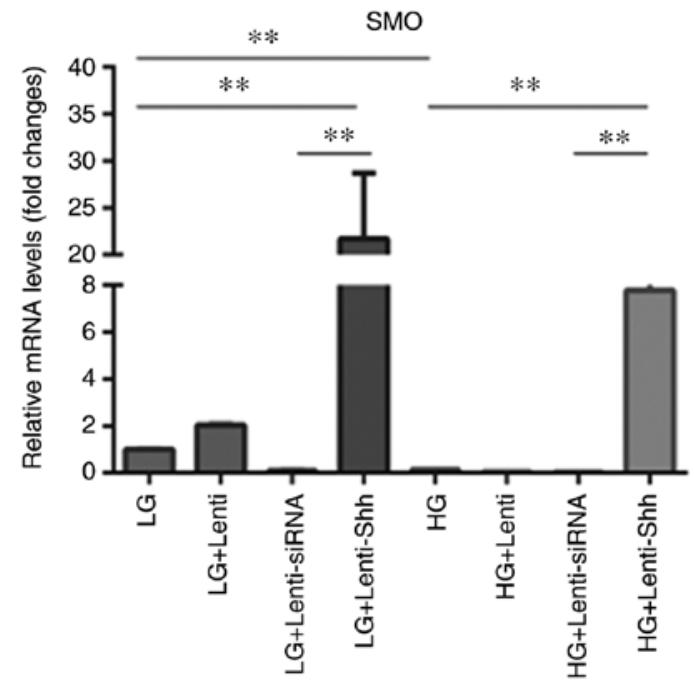

Figure 3. Expression levels of SMO in bone marrow stromal stem cells cultured under eight media conditions: LG, LG + Lenti, LG + Lenti-siRNA, LG + Lenti-Shh, HG, HG + Lenti, HG + Lenti-siRNA and HG + Lenti-Shh. ${ }^{* *} \mathrm{P}<0.01$. HG, high glucose; LG, low glucose; Lenti, lentivirus; Shh, Sonic hedgehog; siRNA, small interfering RNA; SMO, smoothened.

(negative control), respectively. As presented in Fig. 2A-C, Shh protein expression was markedly decreased in BMSCs when cultured in HG medium compared with LG medium, which indicated that HG inhibited Shh protein expression in BMSCs. In addition, Lenti-Shh treatment significantly upregulated Shh expression in LG and HG medium compared with the corresponding controls. These results suggested that following transduction with Lenti-Shh, the decreased expression of Shh in BMSCs under HG conditions could be reversed.

Effect of HG on the expression levels of SMO in BMSCs. Hh signaling in vertebrates is initiated by the binding of secreted Hh proteins to the twelve-transmembrane protein $\mathrm{PTCH}$, which typically suppresses the activity of the seven-transmembrane protein SMO (14). SMO in turn promotes the expression of $\mathrm{Hh}$ target genes by the Gli family of transcription factors and activates Hh signaling (15). The present study performed RT-qPCR in order to quantify the expression levels of SMO (Fig. 3), 

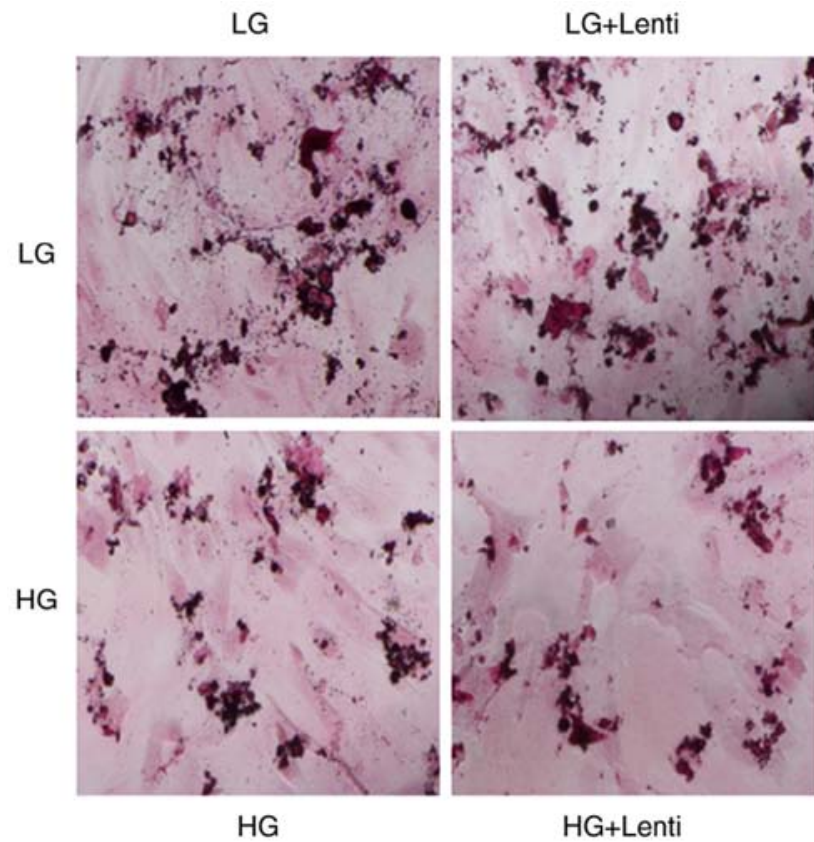

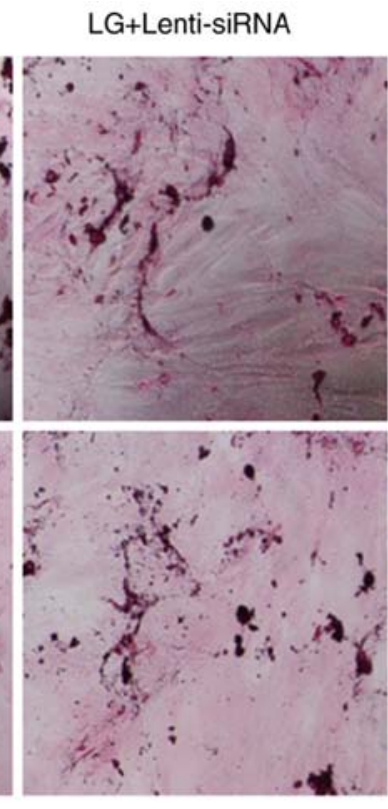

HG+Lenti-siRNA

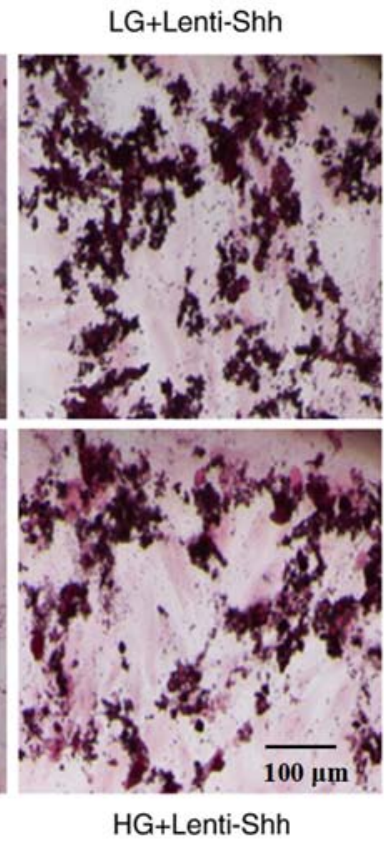

Figure 4. Extent of matrix mineralization in bone marrow stromal stem cells as determined by Alizarin red S staining cultured under eight different media conditions: LG, LG + Lenti, LG + Lenti-siRNA, LG + Lenti-Shh, HG, HG + Lenti, HG + Lenti-siRNA and HG + Lenti-Shh. Scale bar, 100 mm. HG, high glucose; LG, low glucose; Lenti, lentivirus; Shh, Sonic hedgehog; siRNA, small interfering RNA.

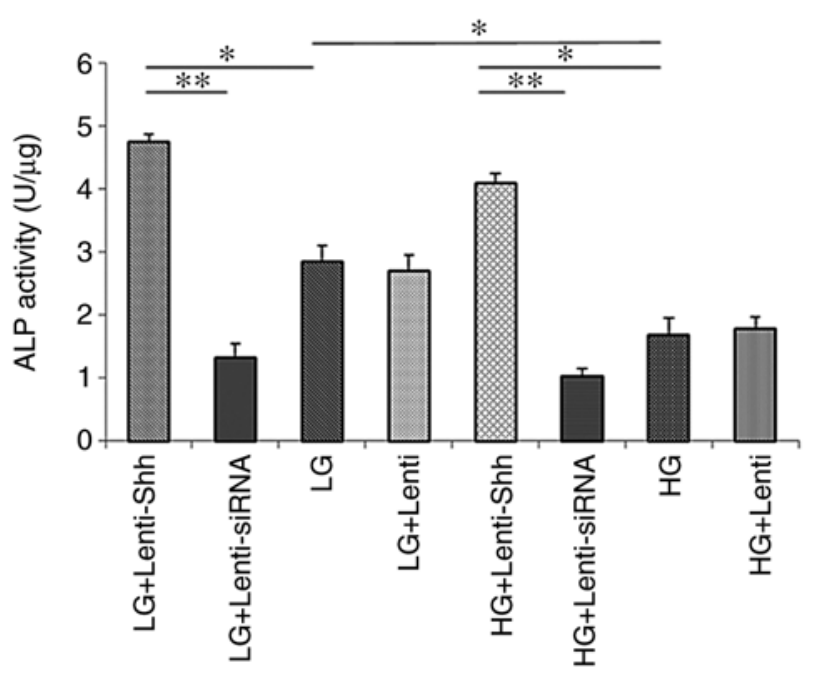

Figure 5. ALP activity of bone marrow stromal stem cellscultured in eight media conditions: LG + Lenti-Shh, LG + Lenti-siRNA, LG, LG + Lenti, $\mathrm{HG}+$ Lenti-Shh, HG + Lenti-siRNA, HG and HG + Lenti was analyzed on day $14 .{ }^{*} \mathrm{P}<0.05,{ }^{* *} \mathrm{P}<0.01$. ALP, alkaline phosphatase activity; HG, high glucose; LG, low glucose; Lenti, lentivirus; Shh, Sonic hedgehog; siRNA, small interfering RNA.

and the results indicated that SMO expression was significantly decreased in the HG group compared with LG group. Following transduction with Lenti-Shh, the LG + Lenti-Shh and $\mathrm{HG}+$ Lenti-Shh groups exhibited significantly upregulated SMO expression compared with the corresponding controls. However, the levels of SMO were decreased in the LG + Lenti-siRNA and HG + Lenti-siRNA groups; the levels SMO expression in the LG + Lenti and HG + Lenti control groups were similar to that of the LG and HG groups, respectively. These results suggested that HG levels inhibited the expression of SMO; however, following transduction with Lenti-Shh, the decreased expression of SMO in BMSCs under HG conditions could be reversed.

Effect of high glucose and Shh pathway on matrix mineralization of BMSCs. Osteoblast-secreted matrix is mineralized into nodules (27). In the present study immunohistochemical staining of mineralized nodules with Alizarin red $\mathrm{S}$ after 21 days of culture was performed in 24-well plates. The results presented in Fig. 4 demonstrated that matrix mineralization in the HG group was decreased compared with the LG group. Following infection with Lenti-Shh, a notable increase in mineralized nodules was observed under LG and HG conditions, compared with LG and HG alone, respectively. However, the LG + Lenti-siRNA and HG + Lenti-siRNA groups exhibited markedly decreased matrix mineralization compared with the other groups; the extent of staining in the LG + Lenti and HG + Lenti control groups was similar to that of the LG and HG groups, respectively. Taken together, these results indicated that $\mathrm{HG}$ levels inhibited the matrix mineralization of BMSCs and this negative effect could be reversed by activating the Shh pathway via transduction with Lenti-Shh.

Effects of HG and Shh pathway on ALP activity in BMSCs. The ALP activity levels of BMSCs cultured in eight different media conditions were detected and are presented in Fig. 5. The results demonstrated that the ALP activity in the HG group was significantly reduced compared with the LG group. Following transduction with Lenti-Shh, the significantly increased ALP activity levels were detected under both glucose conditions compared with the LG and HG groups, respectively $(\mathrm{P}<0.05)$. Conversely, the $\mathrm{LG}+$ Lenti-siRNA and $\mathrm{HG}+$ Lenti-siRNA groups exhibited notably decreased ALP 

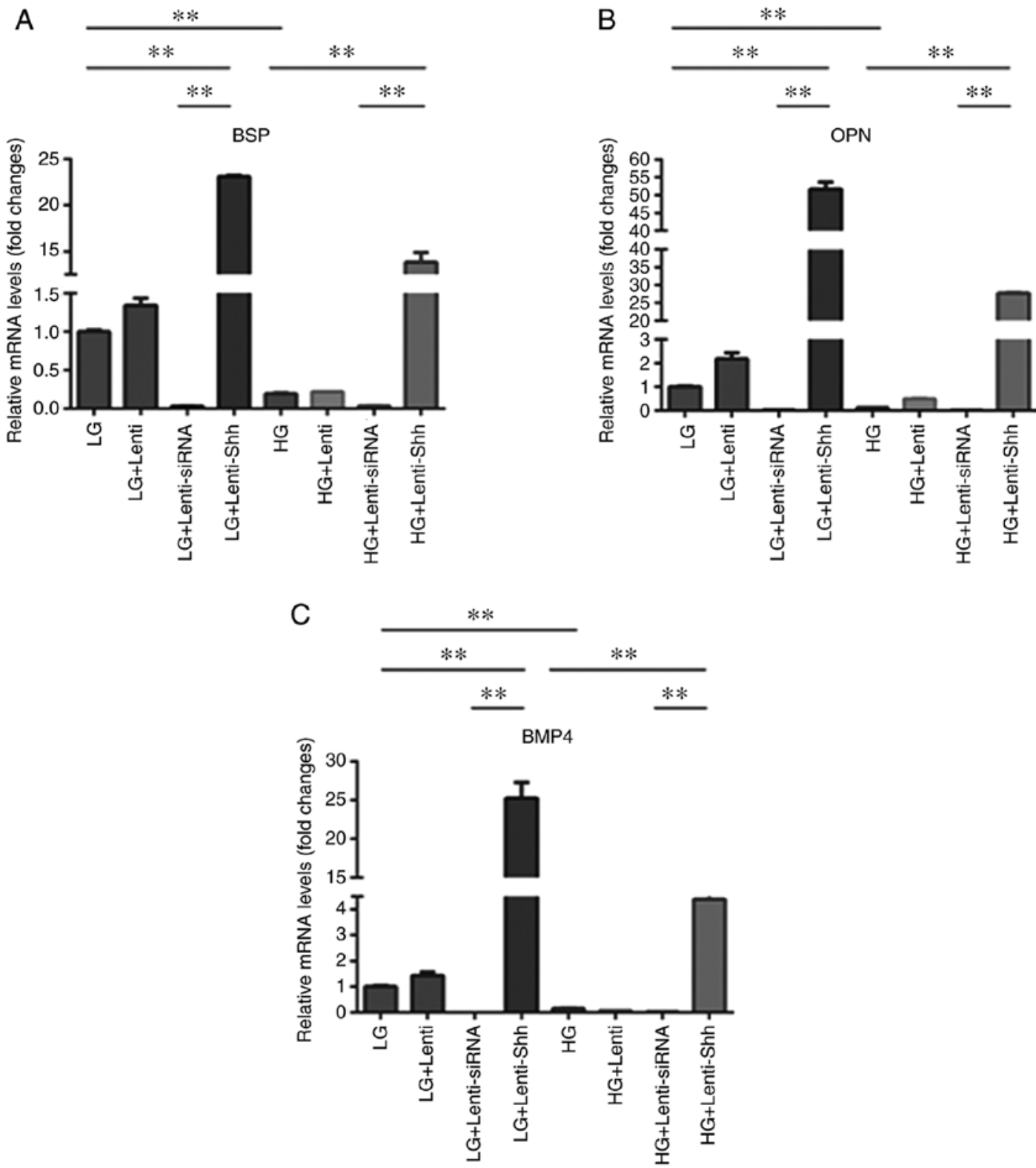

Figure 6. Gene expression of bone marrow stromal stem cells cultured in eight media conditions: LG, LG + Lenti, LG + Lenti-siRNA, LG + Lenti-Shh, HG, $\mathrm{HG}+$ Lenti, HG + Lenti-siRNA and HG + Lenti-Shh. The expression of (A) BSP, (B) OPN and (C) BMP4 was quantified by reverse transcription-quantitative polymerase chain reaction. ${ }^{* *} \mathrm{P}<0.01$. BMP4, bone morphogenic protein 4; BSP, bone sialoprotein; OPN, osteopontin; HG, high glucose; LG, low glucose; Lenti, lentivirus; Shh, Sonic hedgehog; siRNA, small interfering RNA.

activity levels compared with the other groups compared with the corresponding groups under the same glucose conditions. In addition, the ALP activity levels of the LG + Lenti and $\mathrm{HG}+$ Lenti control groups similar to the LG and HG groups, respectively. These results indicated that HG concentrations inhibited the ALP activity of BMSCs and this negative effect could be reversed by activating the Shh pathway via transduction with Lenti-Shh.

Effects of $H G$ on the expression of osteogenesis-related genes in BMSCs and the role of Shh protein. To provide further evidence as to whether a HG inhibits the expression of osteogenesis-related genes in BMSCs and to determine whether Lenti-Shh/siRNA could adjust gene expression, the present study performed RT-qPCR to quantify their relative expression levels. As presented in Fig. 6A-C, the expression levels of bone sialoprotein (BSP), osteopontin (OPN) and BMP4 were significantly downregulated in the HG group compared with the LG group, which indicated that HG inhibited osteogenesis-related gene expression in BMSCs. Following transduction with Lenti-Shh, the LG + Lenti-Shh and HG + Lenti-Shh groups exhibited significantly increased BSP, OPN and BMP4 expression levels compared with the LG and HG groups respectively $(\mathrm{P}<0.05)$. Additionally, the LG + Lenti-siRNA and HG + Lenti-siRNA groups exhibited markedly decreased expression levels compared with the other groups under the same glucose conditions. The expression levels of the aforementioned genes in the LG + Lenti and $\mathrm{HG}+$ Lenti control groups, were similar to those of the LG and HG groups, respectively. These results suggested that the decreased expression of BSP, OPN and BMP4 in BMSCs under HG conditions could be reversed following transduction 
A
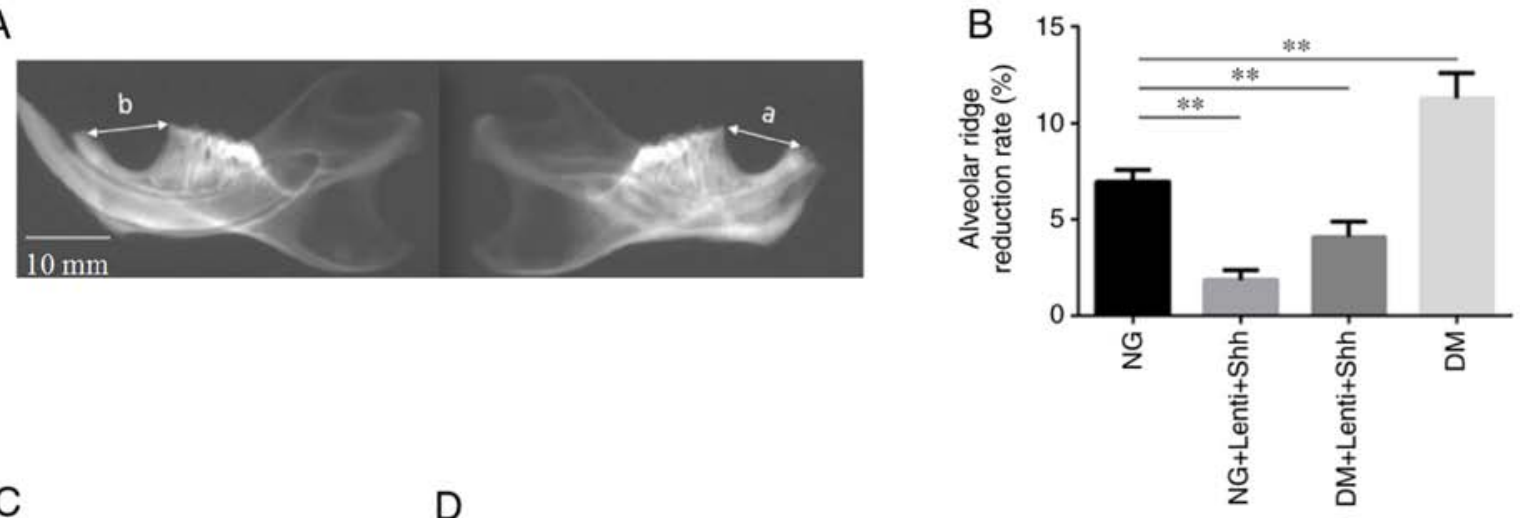

C
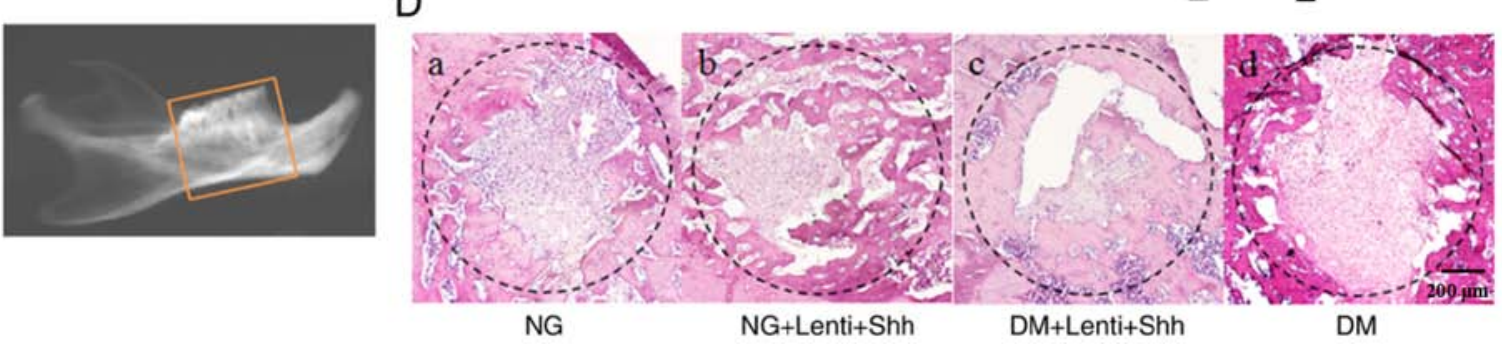

Figure 7. (A) Soft X-ray and histological analyses.The distances between the highest point of the mesial at the first molar to the lingual alveolar ridge margin of the incisor were determined: (a) The length after incisor extraction and (b) the length before incisor extraction. Scale bar, 10 mm. The reduction rate was calculated according to the following formula: (1-a/b) x100\%. (B) The reduction rate results. (C) The orange border area was selected for histological analysis. (D) Hematoxylin and eosin staining: (a) Normal group, (b) normal group with Lenti+Shh, (c) DM group with Lenti+Shh and (d) DM group. Scale bar, $200 \mu \mathrm{m}$. ${ }^{* *} \mathrm{P}<0.01$. DM, diabetes mellitus; NG, normal group; Lenti, lentivirus; Shh, Sonic hedgehog.

with Lenti-Shh. The in vitro experiments were performed in triplicate.

Histological analyses suggests that Lenti-Shh enhances bone formation and reverses the adverse effect of diabetes on tooth sockets in a rat model. The present study sought to confirm whether DM alters the alveolar ridge absorption and the formation of new bones in the tooth socket. The rate of alveolar ridge reduction was measured among the four groups at 8 week (Fig. 7A and B). The results demonstrated that the rate of alveolar ridge reduction was significantly decreased in the normal group compared with the DM group. The reduction rates of the normal group with Lenti-Shh and DM + Lenti-Shh were significantly lower than the normal group. In addition, the histological analysis of the socket revealed that more fibrous connective tissue and reduced new bone formation were detected in the DM group when compared with the normal group (Fig. 7D-a and -d). Importantly, more newly formed bone was detected in the normal group with Lenti-Shh and DM +Lenti-Shh, than in the normal group (Fig. 7D-b and -c). New bone formation from the edge of sockets towards the center and an increased abundance of osteoclasts were evident in the Lenti-Shh treated groups. These data suggest that diabetes inhibited the formation of new bones and that Lenti-Shh may protect alveolar bone, while enhancing new bone formation; thus, increasing the expression of Shh may reverse the negative effect of diabetes.

\section{Discussion}

The present study investigated the effects of HG and the Shh pathway on BMSC differentiation, and the expression of osteogenesis-related genes. From cells transducing with Lenti-Shh and Lenti-siRNA vectors, the results indicated that: i) Shh signaling in osteoblasts was triggered by Lenti-Shh; ii) activation of Shh signaling enhanced the expression of BMP4, BSP and OPN; iii) HGmayimpair osteogenic differentiation; and iv) the inhibitory effects of $\mathrm{HG}$ on the differentiation of BMSCs were reversed via transduction with Shh lentiviral vectors. To verify these observations in vivo, the present study used a rat tooth extraction model, and found that: i) Lenti-Shh treatment resulted in a revealed reductions of alveolar bone to a lesser extent; and ii) significant bone formation inside the extraction socket when compared with the normal and DM groups. Taken together, the results of the present study demonstrated that osteogenic differentiation was affected by the Shh pathway under HG conditions. Furthermore, these effects could be controlled by way of activating Shh signaling.

The Hh signaling pathway serves a key role in numerous processes during embryonic development, as well as the proliferation and differentiation of stem cells, and induces bone formation $(17,28,29)$. Oliveira et al $(30)$ reported that activation of the Hh pathway increased the expression of a panel of genes associated with the osteoblast phenotype development in human mesenchymal stem cells. In addition, the $\mathrm{Hh}$ pathway exerts a pivotal function in driving undifferentiated cells to the osteoblast lineage (30). In addition, several lines of evidence support the notion that activation of the Hh pathway by purmorphamine increases the transcription of numerous genes, including Gli1, PTCH and ALP; however, ALP activity was induced in mouse embryonic mesoderm fibroblasts and pre-osteoblast cell lines $(31,32)$. The results of the present study consistently demonstrated that activation of Shh signaling via 
transduction with Lenti-Shh vectors upregulated the expression of BMP4, BSP and OPN. The present study also observed that the ALP activity and matrix mineralization of BMSCs were increased by triggering Shh signaling. BMP4, BSP and OPN are early transcription factors that are associated with osteoblast differentiation (33). In addition, ALP is involved in the formation and reconstruction of bones (34-36). Therefore the aforementioned bone related genes should be investigated further, in an attempt to provide additional insight into the relationship between the Shh pathway and HG levels.

The incidence of diabetes-associated bone metabolic disorders is increasing (37). If poorly controlled, the quality of life of patients could be severely affected. A previous study demonstrated that patients with T2DM had a 50\% increased mortality and a $21 \%$ increased incidence of hip fracture when compared with individuals without T2DM (38). To a certain extent, our previous findings confirmed that the alveolar bone resorption was more severe in rats with diabetes compared with non-diabetic rats (4). Glucose in the microenvironment markedly affects the gene regulation of cells. A study reported that high levels of glucose inhibits the mRNA expression of ALP and RUNX family transcription factor 2 in periodontal ligament stem cells (39). The results of the present study consistently demonstrated fewer mineralized nodules in the HG groups, as well as ALP activity and the reduced mRNA expression of osteogenic related genes when compared with LG groups. This is in accordance with the findings that in the presence of $\mathrm{HG}$ concentrations, osteoblasts undergo differentiation; however, with a significant delay compared with control- or mannitol-treated cells (40). Additionally, SMO significantly impairs Shh-induced chemotaxis and is accompanied by elevated expression levels of $\mathrm{PTCH}$, which is a protein that inhibits SMO activity (20). In the present study, Shh protein and SMO gene expression were decreased under HG conditions when compared with LG conditions. This may be due to HG-induced downregulation of genes associated with the Shh pathway. In addition the present study observed that, following transduction with Lenti-Shh, the osteoblastic differentiation of BMSCs was promoted, as were the expression of Shh and SMO under HG and LG conditions. As hypothesized, the activation of Shh signaling could prevent HG-mediated BMSCs dysfunction thereby upregulating osteoblast differentiation; however, this beneficial effect was attenuated following transduction with Lenti-siRNA, which was accompanied with a decrease in the expression of bone-related genes, ALP activity and matrix mineralization deposition. As the results of the present study demonstrated the high efficiency of lentiviral-mediated overexpression and downregulation of Shh on osteoblastic differentiation under HG conditions in BMSCs in vitro, future studies should investigate and verify these effects in animal models prior to use in clinical settings. The alveolar ridge reduction rate was significantly higher in DM rats than the NG rats, which indicated reduced bone formation in DM group. Following treatment with Lenti-Shh, a significant loss in alveolar bone was prevented in the NG + Lenti-Shh and DM + Lenti-Shh groups. These results indicated that the activation of Shh signaling could promote bone formation.

Due to its widespread role in embryonic and postnatal development, Shh pathway activators have attracted a great deal of attention as potential therapies (14). The study of Wang et al (41) used an adenovirus that expresses a secreted form of ShhN peptide to activate $\mathrm{Hh}$ signaling in periosteal mesenchymal progenitors and observed robust bone formation. Furthermore, in an additional study, a >90\% transduction efficiency at 1 week was recorded, and continued to demonstrate stable expression for 8 weeks as identified in rat BMSCs transduced with Lenti-CMV-EGFP (42). In the present study, we transduced BMSCs with lentiviral vectors fused with GFP at an MOI of 10 and $>80 \%$ of the cells were GFP positive. Lentiviral vectors were selected as gene delivery vehicles for research due to their capacity to efficiently transduce non-dividing cells, shuttle large genetic payloads and maintain stable long-term transgene expression. It has been reported that Hh signaling could be regulated by the presence of the Shh protein and GANT61 (43). However, proteins and chemicals usually have other side effects on cells. The strong cytotoxicity of GANT61 has a significant effect on cell survival $(44,45)$, which limits its ease of application in animal experiments. An increasing amount of studies investigating bone tissue engineering have investigated the application of cytokines, specific growth factors and artificial scaffolds for promoting bone formation in vivo via transducing signals to modulate cellular activities $(46,47)$. In the present study, the area of new bone formation in the NG + Lenti-Shh and $\mathrm{DM}+$ Lenti-Shh groups was larger than that of the NG and DM groups, respectively. This indicated that Lenti-Shh transgene expression contributed to in vivo bone mineralization and osteogenesis. Thus, lentiviral transgene expression may be considered as an effective therapeutic strategy for overcoming local bone defects, particularly for those with diabetes.

In conclusion, the results of the present study are in agreement with the hypothesis that HG alters the Shh pathway in osteoblasts, which results in defects during osteoblastic differentiation; the activation of Shh signaling could reverse these deleterious effects and promote bone formation. Understanding the mechanism underlying the effects of $\mathrm{HG}$ on osteoblastic differentiation and matrix mineralization deposition may provide novel insight into the possible therapeutic and prevention methods of osteopenia associated with diabetes. Of note, further investigation is warranted to validate the use of Lenti-Shh transgene expression to ensure the safety and efficiency for human use.

\section{Acknowledgements}

Not applicable.

\section{Funding}

The present study was supported by Natural Science Foundation of China (grant no. 81570951), Natural Science Foundation of China (grant no. 81500816), the Innovation Science Foundation of Harbin Medical University (grant no. 2016LCZX10), and Natural Science Foundation of Heilongjiang Province of China (grant no. H2015103).

\section{Availability of data and materials}

The datasets used and/or analyzed during the current study are available from the corresponding author on reasonable request. 


\section{Authors' contributions}

ZLJ designed the study, performed the research, analyzed data, and wrote the paper. BZ designed the in vitro experiments and edited the manuscript. YL designed the in vivo experiments and edited the manuscript. ZLJ, HJ, ZSL, MYL, XFC, YYJ and HDB made substantial contributions to the analysis and interpretation of data, and drafted the manuscript. All authors reviewed the manuscript.

\section{Ethics approval and consent to participate}

All animal experiments were conducted in strict accordance with the principles of medical ethics and were approved by Animal Care and Use Committee of the Second Affiliated Hospital of Harbin Medical University (Harbin, China). All necessary permits were obtained for the described field studies.

\section{Patient consent for publication}

Not applicable.

\section{Competing interests}

The authors declare that there are no competing interests.

\section{References}

1. de Carvalho GB, Dias-Vasconcelos NL, Santos RKF, Brandão-Lima PN, da Silva DG and Pires LV: Effect of different dietary patterns on glycemic control in individuals with type 2 diabetes mellitus: A systematic review. Crit Rev Food Sci Nutr: $1-12,2019$.

2. Marin C, Luyten FP, Van der Schueren B, Kerckhofs G and Vandamme K: The impact of type 2 diabetes on bone fracture healing. Front Endocrinol (Lausanne) 9: 6, 2018.

3. Ge Z, Liu ZZ, Kan J, Zhang JJ, Li SJ, Tian NL, Ye F, Qian XS, Yang S, Chen MX, et al: Stent fracture is associated with a higher mortality in patients with type- 2 diabetes treated by implantation of a second-generation drug-eluting stent. Int J Cardiovasc Imaging 33: 1873-1881, 2017.

4. Kayal RA, Siqueira M, Alblowi J, McLean J, Krothapalli N, Faibish D, Einhorn TA, Gerstenfeld LC and Graves DT: TNF-alpha mediates diabetes-enhanced chondrocyte apoptosis during fracture healing and stimulates chondrocyte apoptosis through FOXO1. J Bone Miner Res 25: 1604-1615, 2010.

5. Liu R, Bal HS, Desta T, Krothapalli N, Alyassi M, Luan Q and Graves DT: Diabetes enhances periodontal bone loss through enhanced resorption and diminished bone formation. J Dent Res 85: 510-514, 2006.

6. Jiang ZL, Cui YQ, Gao R, Li Y, Fu ZC, Zhang B and Guan CC: Study of TNF- $\alpha$, IL-1 $\beta$ and LPS levels in the gingival crevicular fluid of a rat model of diabetes mellitus and periodontitis. Dis Markers 34: 295-304, 2013.

7. Wittrant Y, Gorin Y, Woodruff K, Horn D, Abboud HE, Mohan S and Abboud-Werner SL: High D(+)glucose concentration inhibits RANKL-induced osteoclastogenesis. Bone 42: 1122-1130, 2008.

8. Serrano S, Mariñoso ML, Nacher M, Torres A, Cuevas X, Loreta J, Munné A and Diez A: Modulation of osteoblast activity by serum from diabetic and non-diabetic patients on hemodialysis: A three-dimensional culture study. J Nephrol 17: 369-376, 2004.

9. Liu C and Jiang D: High glucose-induced LIF suppresses osteoblast differentiation via regulating STAT3/SOCS3 signaling. Cytokine 91: 132-139, 2017.

10. Luo J, Sun MH, Kang Q, Peng Y, Jiang W, Luu HH, Luo Q, Park JY, Li Y, Haydon RC and He TC: Gene therapy for bone regeneration. Curr Gene Ther 5: 167-179, 2005.

11. Milone MC and O'Doherty U: Clinical use of lentiviral vectors. Leukemia 32: 1529-1541, 2018.
12. Naldini L, Blömer U, Gallay P, Ory D, Mulligan R, Gage FH, Verma IM and Trono D: In vivo gene delivery and stable transduction of nondividing cells by a lentiviral vector. Science 272 : 263-267, 1996.

13. Naldini L, Blömer U, Gage FH, Trono D and Verma IM: Efficient transfer, integration, and sustained long-term expression of the transgene in adult rat brains injected with a lentiviral vector. Proc Natl Acad Sci USA 93: 11382-11388, 1996.

14. Kimura H, Ng JM and Curran T: Transient inhibition of the Hedgehog pathway in young mice causes permanent defects in bone structure. Cancer Cell 13: 249-260, 2008.

15. Yuan X, Cao J, He X, Serra R, Qu J, Cao X and Yang S: Ciliary IFT80 balances canonical versus non-canonical hedgehog signalling for osteoblast differentiation. Nat Commun 7: 11018-11024, 2016.

16. Wang C, Shan S, Wang C, Wang J, Li J, Hu G, Dai K, Li Q and Zhang X: Mechanical stimulation promote the osteogenic differentiation of bone marrow stromal cells through epigenetic regulation of Sonic Hedgehog. Exp Cell Res 352: 346-356, 2017.

17. Kinto N, Iwamoto M, Enomoto-Iwamoto M, Noji S, Ohuchi H, Yoshioka H, Kataoka H, Wada Y, Yuhao G, Takahashi HE, et al: Fibroblasts expressing Sonic hedgehog induce osteoblast differentiation and ectopic bone formation. FEBS Lett 404: 319-323, 1997.

18. Ho JE, Chung EH, Wall S, Schaffer DV and Healy KE: Immobilized sonic hedgehog N-terminal signaling domain enhances differentiation of bone marrow-derived mesenchymal stem cells. J Biomed Mater Res A 83: 1200-1208, 2007.

19. Liao DM, Ng YK, Tay SSW, Ling EA and Dheen ST: Altered gene expression with abnormal patterning of the telencephalon in embryos of diabetic Albino Swiss mice. Diabetologia 47: 523-531, 2004.

20. Dunaeva M, Voo S, van Oosterhoud C and Waltenberger J: Sonic hedgehog is a potent chemoattractant for human monocytes: Diabetes mellitus inhibits Sonic hedgehog-induced monocyte chemotaxis. Basic Res Cardiol 105: 61-71, 2010.

21. Livak KJ and Schmittgen TD: Analysis of relative gene expression data using real-time quantitative PCR and the 2(-Delta Delta C(T)) method. Methods 25: 402-408, 2001.

22. Srinivasan K, Viswanad B, Asrat L, Kaul CL and Ramarao P: Combination of high fat diet-fed and low-dose streptozotocintreated rat: A model for type 2 diabetes and pharmacological screening. Pharmacol Res 52: 313-320, 2005.

23. Ti Y, Xie GL, Wang ZH, Bi XL, Ding WY, Wang J, Jiang GH, $\mathrm{Bu}$ PL, Zhang Y, Zhong $\mathrm{M}$ and Zhang W: TRB3 gene silencing alleviates diabetic cardiomyopathy in a type 2 diabetic rat model. Diabetes 60: 2963-2974, 2011.

24. Li C, Shi C, Kim J, Chen Y, Ni S, Jiang L, Zheng C, Li D, Hou J, Taichman RS and Sun H: Erythropoietin promotes bone formation through Ephr inB2/EphB4 signaling. J Dent Res 94: 455-463, 2015.

25. Elsubeihi ES and Heersche JN: Quantitative assessment of post-extraction healing and alveolar ridge remodelling of the mandible in female rats. Arch Oral Biol 49: 401-412, 2004.

26. Arai Y, Aoki K, Shimizu Y, Tabata Y, Ono T, Murali R, Mise-Omata S and Wakabayashi N: Peptide-induced de novo bone formation after tooth extraction prevents alveolar bone loss in a murine tooth extraction model. Eur J Pharmacol 782: 89-97, 2016.

27. Reseland JE, Syversen U, Bakke I, Qvigstad G, Eide LG, Hjertner O, Gordeladze JO and Drevon CA: Leptin is expressed in and secreted from primary cultures of human osteoblasts and promotes bone mineralization. J Bone Miner Res 16: 1426-1433, 2001 .

28. Ingham PW and McMahon AP: Hedgehog signaling in animal development: Paradigms and principles. Genes Dev 15: 3059-3087, 2001.

29. McMahon AP, Ingham PW and Tabin CJ: Developmental roles and clinical significance of hedgehog signaling. Curr Top Dev Biol 53: 1-114, 2003.

30. Oliveira FS, Bellesini LS, Defino HL, da Silva Herrero CF, Beloti MM and Rosa AL: Hedgehog signaling and osteoblast gene expression are regulated by purmorphamine in human mesenchymal stem cells. J Cell Biochem 113: 204-208, 2012.

31. Wu X, Walker J,Zhang J, Ding S and Schultz PG: Purmorphamine induces osteogenesis by activation of the hedgehog signaling pathway. Chem Biol 11: 1229-1238, 2004.

32. Sinha S and Chen JK: Purmorphamine activates the hedgehog pathway by targeting smoothened. Nat Chem Biol 2: 29-30, 2006. 
33. Kasai T, Bandow K, Suzuki H, Chiba N, Kakimoto K, Ohnishi T, Kawamoto S, Nagaoka E and Matsuguchi T: Osteoblast differentiation is functionally associated with decreased AMP kinase activity. J Cell Physiol 221: 740-749, 2009.

34. Jung WW: Protective effect of apigenin against oxidative stress-induced damage in osteoblastic cells. Int J Mol Med 33: 1327-1334, 2014.

35. Suh KS, Rhee SY, Jung WW, Kim NJ, Jang YP, Kim HJ, Kim MK, Choi YK and Kim YS: Chrysanthemum zawadskii extract protects osteoblastic cells from highly reducing sugar-induced oxidative damage. Int J Mol Med 32: 241-250, 2013.

36. Kawai $S$ and Sugiura T: Characterization of human bone morphogenetic protein (BMP)-4 and -7 gene promoters: Activation of BMP promoters by Gli, a sonic hedgehog mediator. Bone 29: 54-61, 2001.

37. Courties A, Berenbaum F and Sellam J: The phenotypic approach to osteoarthritis: A look at metabolic syndrome-associated osteoarthritis. Joint Bone Spine: Dec 22, 2018 doi: 10.1016/j. jbspin.2018.12.005 (Epub ahead of print).

38. Tebé C, Martinez-Laguna D, Moreno V, Cooper C, Diez-Perez A Collins GS and Prieto-Alhambra D: Differential mortality and the excess rates of hip fracture associated with type 2 diabetes: Accounting for competing risks in fracture prediction matters. J Bone Miner Res 33: 1417-1421, 2018.

39. Kim SY, Lee JY, Park YD, Kang KL, Lee JC and Heo JS Hesperetin alleviates the inhibitory effects of high glucose on the osteoblastic differentiation of periodontal ligament stem cells. PLoS One 8: e67504, 2013.

40. Balint E, Szabo P, Marshall CF and Sprague SM: Glucose-induced inhibition of in vitro bone mineralization. Bone 28: 21-28, 2001
41. Wang Q, Huang C, Zeng F, Xue M and Zhang X: Activation of the Hh pathway in periosteum-derived mesenchymal stem cells induces bone formation in vivo: Implication for postnatal bone repair. Am J Pathol 177: 3100-3111, 2010.

42. Sugiyama O, An DS, Kung SP, Feeley BT, Gamradt S, Liu NQ, Chen IS and Lieberman JR: Lentivirus-mediated gene transfer induces long-term transgene expression of BMP-2 in vitro and new bone formation in vivo. Mol Ther 11: 390-398, 2005.

43. Guan CC, Yan M, Jiang XQ, Zhang P, Zhang XL, Li J, Ye DX and Zhang FQ: Sonic hedgehog alleviates the inhibitory effects of high glucose on the osteoblastic differentiation of bone marrow stromal cells. Bone 45: 1146-1152, 2009.

44. Mazumdar T, DeVecchio J, Shi T, Jones J, Agyeman A and Houghton JA: Hedgehog signaling drives cellular survival in human colon carcinoma cells. Cancer Res 71: 1092-1102, 2011.

45. Pan D, Li Y, Li Z, Wang P and Liang Y: Gli inhibitor GANT61 causes apoptosis in myeloid leukemia cells and acts in synergy with rapamycin. Leuk Res 36: 742-748, 2012.

46. Lee SJ: Cytokine delivery and tissue engineering. Yonsei Med J 41: 704-719, 2000.

47. Marei MK, Nouh SR, Saad MM and Ismail NS: Preservation and regeneration of alveolar bone by tissue-engineered implants. Tissue Eng 11: 751-767, 2005.

This work is licensed under a Creative Commons Attribution-NonCommercial-NoDerivatives 4.0 International (CC BY-NC-ND 4.0) License. 